\title{
EDITORIAL
}

\section{Inequality in health: a question with many answers}

Inequality in health is a key topic in health economics. It is, however, hard to capture in terms of scalar measures because inequality can be defined in a number of ways. Even if we restrict ourselves to income-related inequality in health, which is commonly focused on in health economics, it is difficult to provide clear-cut answers to policy-makers about the current situation. This is because of uncertain parameters arbitrarily embedded in, for example, the popular relative concentration index (Erreygers et al., 2012, Kjellsson and Gerdtham, 2013, Kjellsson et al., 2015).

This does not matter much if the aim is only to evaluate whether inequality exists or not. In such a case, it suffices to eyeball a bar chart showing the health distribution over income, since there is no apparent need to aggregate into an index value. However, this question is seldom of major interest because inequality, as such, is generally the default hypothesis. One of few examples where evidence for inequality is hard to establish is the period before the industrial revolution (Harris, 2004). In modern times, inequality is more a rule than an exception, regardless of whether one considers trivial or serious health conditions, or whether one considers privately or publicly financed and driven health systems.

A greater challenge is to capture whether the inequality moves over time and space. This is a far more complex question. Here, index tools play a larger role, but the results may depend on the chosen measure. Indeed, because there is an infinite number of variants of the concentration index, due to arbitrary weighting of health across income rank, the same analyst can arrive at more than one answer about the current inequality situation. From the perspective of policy, this is at least one answer too many.

Although the issue above of measurement of inequality has been sorted out somehow, challenging issues remain about why the measured inequality moves over time and space, whether this depends on supply or demand, and whether individual preferences (choices) can be distinguished from discrimination (bias). These issues impose high methodological and data requirements. In the Nordic countries, we have advantages over many other countries, because of administrative register data on deaths, income and education, etc. that can also be merged and integrated with rich survey data. Although, these data are useful, and are extensively used, there remains a limit as to what questions they can answer. For example, we may observe inequality in outpatient doctor visits. After controlling extensively for age, sex and "need" factors, significant income-related inequality may remain. Since we can never rule out the missing pieces, the question of whether the inequality reflects discrimination or preferences remains unanswered. Thus, when the limit of observational data analysis has been reached, the discussion about underlying mechanisms that may have driven the findings is left to speculation.

Behavioral economics and experiments can contribute to research on health inequality and complement observational data analysis, but use of these methods remains very limited in economic analysis of health inequality. Experiments could be used to shed light on the many missing pieces indicated above, for example how inequality measures should be constructed, based on how individuals experience different equivalence criteria about what changes an index measure should capture. Experiments can also potentially be used to distinguish between discrimination and choice, for example in the above-mentioned inequality in outpatient doctor visits. By shedding new light on what individuals value most 
when it comes to measuring (in)equality, and why certain inequalities come about, behavioral economics and experiments may be able to provide insights into how inequality in health can be reduced. In doing so, they could help take research on inequality in health to the next level.

Ulf-G. Gerdtham

\section{References}

Erreygers, G, Clarke, P, Van Ourti, T. (2012). Mirror, mirror, on the wall, who in this land is fairest of all?" - Distributional sensitivity in the measurement of socioeconomic inequality of health. Journal of Health Economics 31; 257-270.

Harris, B. (2004). Public health, nutrition, and the de- cline of mortality: The McKeown thesis revisited, Social History of Medicine 17(3): 379-407.

Kjellsson, G., Gerdtham, U-G. and Petrie, D. (2015). Lies, damned lies, and health inequality measurements: Understanding the value judgments. Epidemiology. 26(5):673-80.

Kjellsson, G. and Gerdtham, U-G. (2013). On correcting the concentration index for binary variables. Journal of health economics. 32(3):659-70. 